\title{
Mode Identification of the $\beta$ Cephei star BW Vulpeculae ${ }^{1}$
}

\author{
C. Aerts ${ }^{2}$, T. Van Hoolst, and P. Mathias ${ }^{3}$ \\ Instituut voor Sterrenkunde, Katholieke Universiteit Leuven, \\ Celestijnenlaan 200 B, B-3001 Heverlee, Belgium
}

Abstract. We identify the pulsation mode of BW Vul by means of both the moment method and the method of photometric amplitudes and find a radial pulsation. We briefly study the non-linear behaviour of $\mathrm{BW}$ Vul.

\section{Introduction}

BW Vul (HD 199140) is a $\beta$ Cephei star of which the radial-velocity (RV) curve has an extremely large amplitude $(200 \mathrm{~km} / \mathrm{s}$ peak-to-peak) and shows a velocity still-stand. A detailed spectroscopic analysis of BW Vul is presented by Furenlid et al. (1987), while Moskalik \& Buchler (1994) studied hydrodynamical models for BW Vul and found that the star pulsates in a fundamental mode. Both studies are based on the assumption that BW Vul exhibits a radial pulsation, although existing mode identifications point towards a non-radial mode $(\ell=$ 2, $m=-1$ : Kubiak 1978, $\ell=-m=2$ : Odell 1981).

\section{Observations}

Our 97 spectra were obtained with the CFHT f8.2 Coudé Spectrograph on July $10-13,1992$. A resolving power of 30,000 , a $\mathrm{S} / \mathrm{N}$ ratio of 250 , and a time resolution of $\mathrm{P} / 30(\mathrm{P}=4 \mathrm{~h} 49 \mathrm{~min})$ was obtained. The region of the spectrum centred around the SiIII triplet $(\lambda \lambda 4553,4568,4575)$ was observed. Twenty-four multicolour observations in the Geneva system, spread over 10 years, were kindly put at our disposal by the Observatoire de Genève.

\section{Mode identification}

The question has arisen if the harmonics in the light-curve of BW Vul (van der Linden \& Sterken 1987) are due to a non-linear effect of one mode or to the presence of different modes. Both the multicolour photometry and the variation

\footnotetext{
${ }^{1}$ Based on observations obtained with the CFHT, Hawaii

${ }^{2}$ Senior Research Assistant, Belgian National Fund for Scientific Research

${ }^{3}$ Present Address: Observatoire de la Côte d'Azur, Département Fresnel, BP 229, F-06304 Nice Cedex 4, France
} 
of the line parameters (RV, EW, FWHM) indicate that the relation between the amplitudes of the variations with $f=4.974 \mathrm{c} / \mathrm{d}$ and with its harmonics is the same. This is not what we expect if different modes would be present, since they would have a different signature in, e.g., the RV compared to the FWHM, and also in the colours. We thus prefer a mono-periodic pulsation model for BW Vul.

The mode is identified by means of the moment method (Aerts et al. 1992), from which we find a radial pulsation with an amplitude of $90 \mathrm{~km} / \mathrm{s}$. This result is confirmed by an analysis with the method of photometric amplitudes (Heynderickx et al. 1994), from which we also find that non-adiabatic effects are important in BW Vul. We thus contradict earlier identifications and find a result that is fully consistent with the studies by Furenlid et al. (1987) and by Moskalik \& Buchler (1994).

\section{Non-linear pulsations}

In order to quantify the non-linear effects of BW Vul, we have calculated a RV curve for a non-linear radial pulsation in case of BW Vul by means of amplitude equations. Our theoretical curve shows no velocity still-stand, and the amplitudes of the terms varying with the harmonics are much smaller than the corresponding observed ones. Possible explanations are that we have used an adiabatic theory, that shocks are not taken into account with amplitude equations, and that the radiative transfer in the atmospheric layers was not taken into account.

\section{Conclusions}

We have found that BW Vul pulsates in a radial mode, that its non-adiabatic effects are important, and that its non-linear effects are large and cannot be treated by means of amplitude equations in the adiabatic approximation. We plan to study our line profiles in more detail by means of a non-adiabatic, nonlinear pulsation theory in combination with hydrodynamical models.

Full details of this study will be published elsewhere (submitted to A\&A).

\section{References}

Aerts, C., De Pauw, M., \& Waelkens, C. 1992, A\&A, 266, 294

Furenlid, I., et al., 1987, ApJ, 319, 264

Heynderickx, D., Waelkens, C., \& Smeyers, P. 1994, A\&AS, 105, 447

Kubiak, M. 1978, Acta Astron., 28, 153

Moskalik, P., \& Buchler, J.R. 1994, in Pulsation, Rotation and Mass Loss in Early-Type Stars, eds. L.A. Balona, H.F. Henrichs \& J.M. LeContel, Kluwer Academisch Publishers, p.19

Odell, A.P. 1981, ApJ, 246, L77

van der Linden, D., \& Sterken, C. 1987, A\&A, 186, 129 